\title{
Pourquoi deux Centres de Brûlés adultes en Suisse, dont un au CHUV?
}

\author{
M. M. Berger ${ }^{a}$, W. Raffoul ${ }^{b}$, P. Maravic ${ }^{a}$, V. Séchet ${ }^{b}$, R. Chioléro $^{a}$
}

Le nombre de patients brûlés graves est resté stable au cours de la dernière décennie. Ces patients nécessitent une prise en charge très spécialisée dans un centre dédié. La Suisse dispose de deux centres pour patients adultes au CHUV à Lausanne, et à l'Hôpital Universitaire de Zurich.

L'article décrit les critères d'admission, les traitements spécialisés appliqués dans les centres, ainsi que la filière de la prise en charge jusqu'à la réhabilitation. Selon les critères internationaux, deux centres spécialisés sont pleinement justifiés en Suisse par la taille de la population, les caractéristiques linguistiques et culturelles, ainsi que par le découpage géographique du pays.

$\begin{array}{ll}\text { Abbréviations } \\ \text { ABA } & \begin{array}{l}\text { American Burn } \\ \text { Association }\end{array} \\ \text { BSA } & \begin{array}{l}\text { Body surface area = } \\ \text { Surface corporelle } \\ \text { totale }\end{array} \\ \text { CPR } \quad \begin{array}{l}\text { Service de Chirurgie } \\ \text { Plastique et } \\ \text { Reconstructive }\end{array} \\ \text { SIC } \quad \begin{array}{l}\text { Soins Intensifs } \\ \text { Chirurgicaux } \\ \text { Unité brûlés } \\ \text { des SIC }\end{array} \\ \text { SIC-CB }\end{array}$

a Soins Intensifs Chirurgicaux et Centre des Brûlés, CHUV

b Service de Chirurgie Plastique et Reconstructive

Correspondance: Mette M. Berger Soins Intensifs Chirurgicaux et Centre des Brûlés CHUV - BH 08.660 CH-1011 Lausanne Tél. 0213142095

\section{Introduction}

En Suisse environ 130 à 150 brûlés graves adultes sont traités chaque année dans les deux centres spécialisés de Lausanne et Zurich. La prévalence des brûlures a certes globalement diminué dans les pays occidentaux au cours des dernières décennies grâce aux mesures de prévention, mais le nombre de brûlés graves est stable [1]. Les victimes sont souvent jeunes. Les causes les plus fréquentes de ces brûlures sont des accidents domestiques, de loisirs et de travail, ainsi que des immolations.

Au total 10 à 11 lits adultes sont actuellement exploités dans les 2 centres de brûlés suisses: 5 au CHUV de Lausanne répartis entre les Soins Intensifs (3 lits) et l'étage, et 5 à 6 lits à Zurich (dont 3 à 4 de soins intensifs). Le Kantonsspital de Bâle (qui n'est pas un centre tel que décrit plus bas) peut traiter 1 à 2 brûlés de gravité modérée. Selon les chiffres de l'OMS [2], la Suisse se situait en 1993 parmi les 10 pays les plus faiblement dotés en lits brûlés avec 3,5 lits par million d'habitants pour une moyenne internationale de 8,7 lits/ million d'habitants. Depuis le nombre des lits spécifiques a été réduit. Notre pays ne dispose plus que de 2 lits par million d'habitants et se trouve au même rang que l'Autriche (2 lits/ million) et la Finlande (2,4 lits/million). Cette situation est préoccupante puisque nous ne pouvons pas compter sans autres sur les pays voisins pour prendre en charge les brûlés qui excèderaient la capacité de nos centres. Les récents évènements en Suisse (tunnel du Gothard 2001) et à l'étranger (attentat de Bali 2002) montrent à l'évidence la grande précarité des ressources disponibles. Onze morts ont été recensés lors de l'incendie du Gothard: si ces victimes avaient survécu, elles auraient nécessité une prise en charge qui aurait largement dépassé nos capacités, comme l'a confirmé la crise liée au rapatriement des 2 victimes Suisses de l'attentat de Bali.

La fermeture des lits spécialisés a conduit à un déficit de lits particulièrement marqué dans notre pays et en France voisine, où plusieurs centres ont été fermés, qui auparavant traitaient les brûlés (exemple l'Hôpital Rothschild à Paris). Il en résulte une surcharge chronique des centres restants. Le CHUV est sollicité lorsque les 2 centres spécialisés de Lyon sont débordés. Ces 2 centres ont aussi été contraints de refuser des patients (85 refus lors du premier semestre 2003 communication personnelle des médecins responsables). Dans ce cas, les patients admis au CHUV sont re-transférés en France, souvent après plusieurs semaines de traitement. Les difficultés de nos voisins se reflètent par l'augmentation des admissions depuis l'étranger au CHUV jusqu'en 2002 (tab. 1). En 2003, le CHUV a dû refuser ces transferts pour pouvoir traiter les patients de notre région, faute de moyens. En raison des limites de nos ressources internes (temps opératoire et disponibilité anesthésique), il a été plus difficile de faire face aux demandes d'admission, en particulier en période de pointe: fin octobre 2003, notre centre avait déjà refusé 14 brûlés pour un total de 29 patients traités en soins intensifs.

A une période où les priorités sanitaires sont rediscutées au niveau fédéral et cantonal, cet article a pour objectif de présenter les spécificités des brûlés et les prestations offertes par des centres spécialisés, de démontrer la nécessité du maintien de 2 centres en Suisse malgré le contexte médico-économique difficile.

\section{Pourquoi des centres spécialisés pour les brûlés graves?}

L'existence de centres spécialisés fait l'objet d'un consensus international depuis les années 70 $[3,4]$. Les brûlures étendues et profondes sont parmi les lésions accidentelles les plus graves: elles nécessitent une prise en charge complexe et 
Tableau 1

Caractéristiques des patients admis au CHUV depuis 1995, avec indication du nombre total d'adultes traités à Zurich.

\begin{tabular}{|c|c|c|c|c|c|c|c|c|c|}
\hline Activité & 1995 & 1996 & 1997 & 1998 & 1999 & 2000 & 2001 & 2002 & 30 juin 2003 \\
\hline Admissions brûlés SIC-CB & 36 & 40 & 37 & 29 & 39 & 42 & 30 & 24 & 19 \\
\hline - dont enfants & 6 & 5 & 2 & 8 & 11 & 4 & 10 & 8 & 6 \\
\hline Admissions assimilés & nd & nd & nd & 0 & 1 & 4 & 3 & 3 & 1 \\
\hline Patients brûlés CPR* & nd & nd & nd & 36 & nd & 18 & 14 & 16 & 8 \\
\hline Surface brûlée moyenne (\% BSA) & 21 & 24 & 25 & 24 & 23 & 31 & 30 & 33 & 34 \\
\hline \multicolumn{10}{|l|}{ Provenance } \\
\hline$-V D$ & 12 & 22 & 17 & 11 & 17 & 18 & 11 & 11 & nd \\
\hline$-G E$ & 13 & 3 & 6 & 6 & 12 & 6 & 4 & 3 & nd \\
\hline - Romandie autre & 10 & 10 & 9 & 8 & 6 & 11 & 8 & 6 & nd \\
\hline - Suisse autre & 0 & 2 & 2 & 2 & 2 & 0 & 0 & 0 & nd \\
\hline - Etranger & 1 & 2 & 3 & 2 & 2 & 4 & 6 & 7 & nd \\
\hline Total patients brûlés traités au CHUV & nd & nd & nd & 65 & nd & 68 & 57 & 50 & 28 \\
\hline Refus d'admission & nd & nd & nd & nd & nd & 0 & 7 & 11 & 5 \\
\hline Total patients Zurich [1] & 76 & 67 & 74 & 79 & 47 & 81 & 99 & 100 & nd \\
\hline
\end{tabular}

particulièrement lourde en terme de soins. La gravité des brûlures dépend de la surface corporelle atteinte (exprimée en \% de la surface corporelle totale, ou BSA), de la profondeur des brûlures et de l'âge du patient. En l'absence de soins spécialisés, les brûlés ont une cicatrisation pathologique, une récupération fonctionnelle de moindre qualité, et une surmortalité. Au plan médico-économique le traitement en centre de brûlés est plus efficace (cost effectiveness élevé) avec un séjour hospitalier plus court [5]. L'admission dans un centre suit des critères précis (tab. 2). Les patients présentant des brûlures modérées peuvent être traités dans des hôpitaux régionaux, alors que les brûlures les plus légères et les plus fréquentes sont traitées ambulatoirement.

Un nombre limité de patients non brûlés souffrant d'affections cutanées «assimilables» aux brûlures bénéficient également de ces centres. Il s'agit des fascéites nécrosantes, d'affections der-

Tableau 2

Critères d'admission en Centre spécialisé de I'American Burn Association.

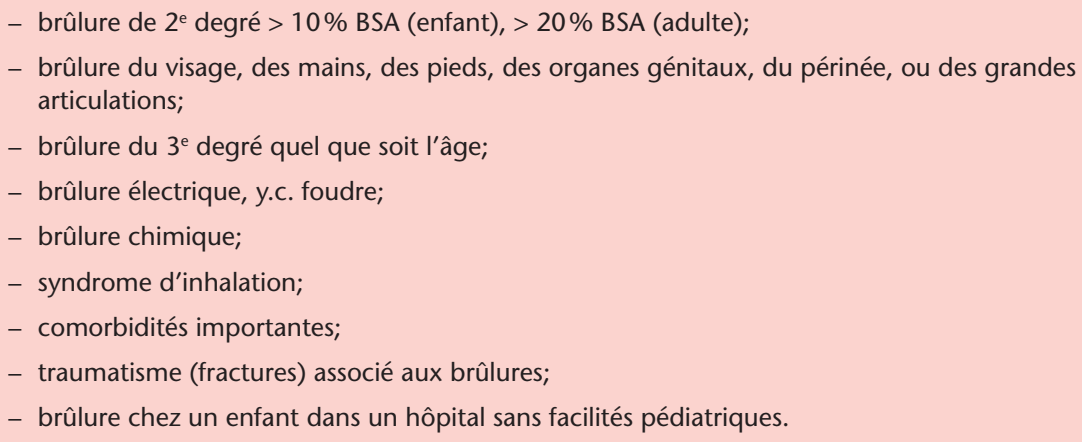

matologiques telles que le syndrome de Lyell (toxic epidermal necrolysis) et autres dermatoses étendues [6, 7].

Un brûlé grave requiert une prise en charge multidisciplinaire dès son admission (fig. 1). Elle nécessite la présence de nombreux spécialistes: 1) une équipe infirmière spécialisée; 2) une équipe médicale de soins intensifs, de chirurgie plastique, et d'anesthésie dédiée; 3) des médecins nutritionnistes; 4 ) des physiothérapeutes et ergothérapeutes; 5) des psychiatres; 6) des médecins consultants multiples: ORL, gastro-entérologues, infectiologues, pneumologues, radiologues, etc.; 7) des biologistes maîtrisant la culture cellulaire (kératinocytes, fibroblastes); 8) des orthopédiste-bandagistes (pansements compressifs, etc.); et 9) des spécialistes de la rééducation. Il faut disposer de boxes de soins intensifs adaptés (surface supérieure aux dimensions habituelles, chambres à $25-30^{\circ} \mathrm{C}$ et à humidité élevée), d'une salle d'opération dédiée, d'une douche, et de lits d'hospitalisation. Une telle constellation de moyens existe au CHUV pour les brûlés adultes, alors qu'elle est actuellement incomplète pour les enfants brûlés, pour qui il existe en Suisse un seul centre vraiment spécialisé au Kinderspital de Zurich.

Les traitements chirurgicaux ont beaucoup évolué au cours des 10 dernières années avec l'apparition de nouveaux substituts cutanés artificiels (fig. 2), mais aussi avec le développement de cultures de cellules multicouches incluant kératinocytes et fibroblastes. Ces techniques, qui s'appliquent surtout aux patients brûlés sur plus de $40 \%$ de la surface corporelle, requièrent un 
ensemble de compétences étendues, incluant des biologistes maîtrisant les cultures cellulaires: le service de Dermatologie du CHUV produit les kératinocytes pour les 2 centres du CHUV et de Zurich [8]. Il faut aussi une équipe médicale capable de maintenir en vie les patients pendant ces périodes de 2 à 3 semaines qui sont requises pour la réalisation de chaque étape des cultures.

L'importance des moyens et des compétences nécessaires à la prise en charge des grands

Figure 1

Spécialités nécessaires à la prise en charge du brûlé grave en soins intensifs: la prise en charge continue est assurée par les infirmières et les médecins de l'unité avec la collaboration d'une multitude des spécialistes.

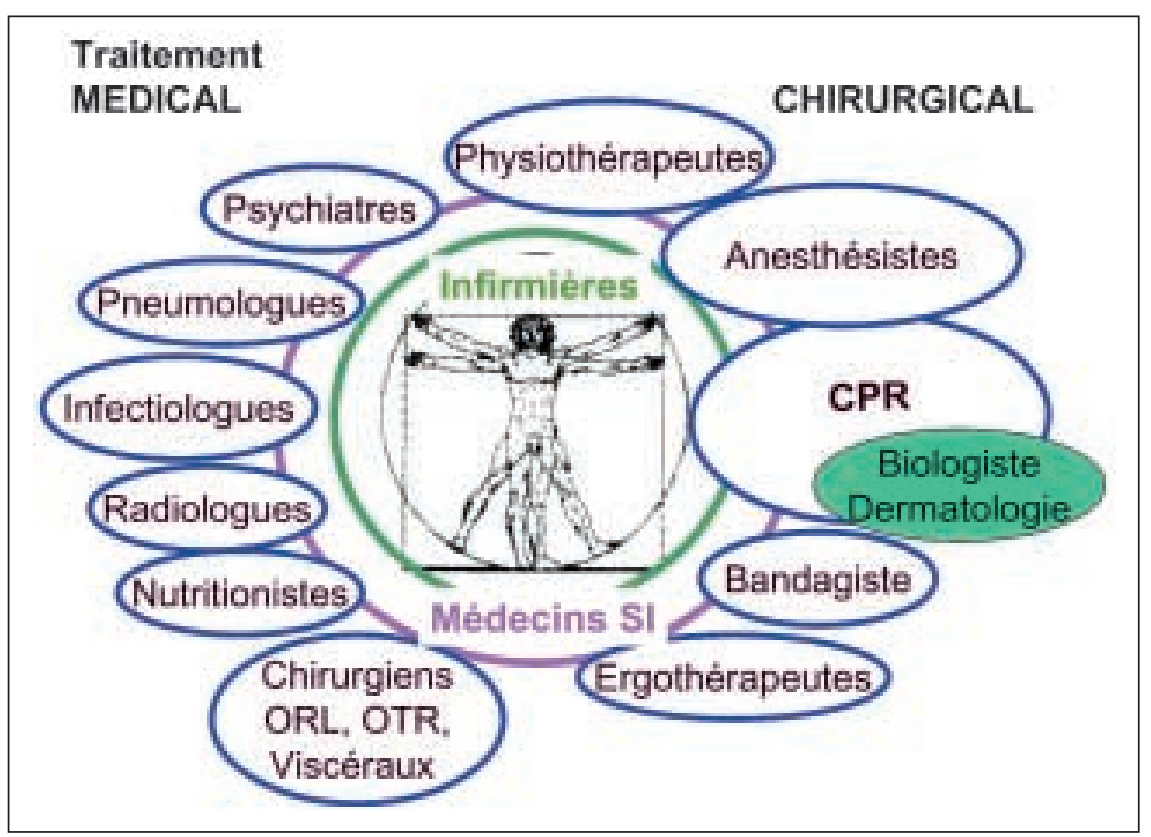

Figure 2

Traitement chirurgical d'une brûlure profonde du bras après débridement, avec le substitut dermique Integra ${ }^{\circledR}$, puis couverture de celui-ci à 2 semaines par des kératinocytes de culture.

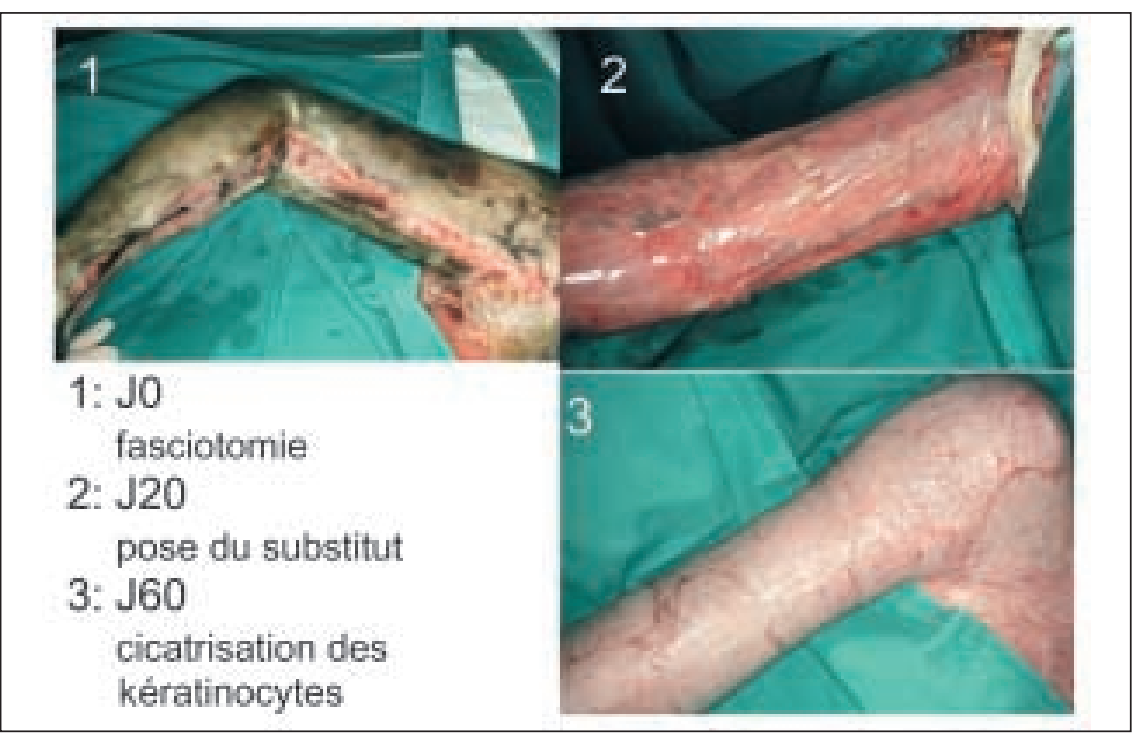

brûlés explique le nombre limité de centres: on admet généralement qu'un centre se justifie pour une population d'environ 3 à 5 millions d'habitants, ce qui implique 2 centres en Suisse, mais pas plus. Qui dit «expert», dit compétences qu'il faut maintenir. La notion de «nombre critique» de patients est particulièrement importante dans la prise en charge des brûlés: le seuil se situe aux environs de 40 patients brûlés graves par an, avec en plus des brûlés moins gravement atteints. Ce nombre permet le développement et le maintien d'un enseignement et d'une recherche spécifiques basés sur les critères d'accréditation de l'American Burn Association (ABA).

\section{Les étapes du traitement du grand brûlé}

Le traitement des brûlés est prolongé (fig. 3): les patients séjournent en moyenne en unité spécialisée 1 à 1,5 jours par pourcentage de surface corporelle brûlée profondes. On peut définir 4 grandes phases de traitement [4]:

1. Une phase initiale d'évaluation et de réanimation, qui dure 24 à 72 heures et vise à maintenir/rétablir l'homéostasie des organes et systèmes [9]. Les techniques de réanimation se sont affinées et visent maintenant à éviter aussi bien les effets de la sur-réanimation que ceux, dévastateurs, de la sous-réanimation [10].

2. Une phase de stabilisation cardio-respiratoire, pendant laquelle les chirurgiens débrident et ferment provisoirement les plaies par des pansements conventionnels et biologiques complexes lors d'interventions sériées sous anesthésie-sédation. Cette phase dure 10 à 21 jours, et requiert des hydrothérapies (douches) et des pansements répétés.

3. Une phase de stabilisation pendant lequel le patient bénéficie toujours d'une réanimation intensive et pendant laquelle les chirurgiens procèdent à la fermeture définitive des plaies par des greffes cutanées permanentes. Elle s'étend de la $2^{\mathrm{e}}$ à la 8 à $10^{\mathrm{e}}$ semaine (parfois plus longtemps pour les très grands brûlés). Il faut assurer une nutrition artificielle prolongée, et de la physiothérapie intensive dans un cadre de soins intensifs lourds [11]. Le risque de complication infectieuse est très élevé, en raison de la perte de la barrière cutanée et de la dépression du système immunitaire.

4. La phase de réhabilitation, de reconstruction et de réintégration qui commence le $1^{\mathrm{er}}$ jour de l'hospitalisation et qui peut durer 2 à 3 ans. 


\section{Pourquoi un Centre Romand des Grands Brûlés (CRGB)?}

La grandeur de la population nécessitant 2 centres spécialisés, comment faut-il les répartir? L'attribution à Lausanne d'un des centres est liée à: 1) l'histoire du centre qui existe officiellement depuis plus de 20 ans; 2) la multi-culturalité et la multi-lingualité Suisse: traiter un brûlé dans sa langue est un atout psychologique important; et enfin 3) le découpage géographique de la Suisse qui rend les déplacements parfois longs (ex: pour un jeune brûlé grave transféré à Zurich en 2002 par manque de place au CHUV, les trajets nécessaires pour chaque visite des proches prenaient $2 \times 4$ heures: l'hospitalisation prolongée a fait «exploser» la famille).

Le CHUV exploite depuis 1982 un véritable centre des brûlés. A l'origine, il s'agissait d'une unité fermée et autonome, exclusivement dédiée aux brûlés, rattachée au service de chirurgie plastique et reconstructrice (CPR). En 1994, le centre est transformé en une unité multi-fonctionnelle traitant également des patients de soins intensifs de chirurgie. Cette mesure a permis de fidéliser le personnel soignant au sein de l'unité et de garantir le développement et le maintien de compétences infirmières et médico-chirurgicales. En 1996, l'unité du Centre des brûlés est reconnue comme unité de soins intensifs [12], qui a été intégralement informatisé en 1999, permettant de renforcer le contrôle de qualité et la recherche [13]. Pour assurer son fonctionnement du centre, il est nécessaire de disposer de moyens considérables, susceptibles de réduire la disponibilité du bloc opératoire, des équipes d'anesthésie et des soins intensifs pour les autres services.

Le centre assure la prise en charge de la majorité des grands brûlés adultes des cantons romands et de quelques patients de France voisine (tab. 1). Il a souvent été dit que le centre du CHUV était plus petit que celui de Zurich. La différence des chiffres officiels jusqu'en 2001 s'explique par le mode de présentation des statistiques lausannoises: seuls les patients admis en soins intensifs (ci-après dénommés SIC-CB) étaient comptabilisés, omettant les patients admis directement en CPR, alors que la totalité des patients étaient inclus dans les statistiques de Zurich [1].

Enfin toute brûlure grave constitue une modification parfois dramatique du schéma corporel. $\mathrm{Au} \mathrm{CHUV,} \mathrm{une} \mathrm{approche} \mathrm{psychologique} \mathrm{spé-}$ cifique a été développée sous l'égide du service de Psychiatrie de Liaison. Pour des cas particulièrement graves, avec douleurs chroniques, les services d'antalgie et de soins palliatifs contribuent également au traitement. Les problèmes psychologiques sont aggravés par des obstacles linguistiques. L'équipe de psychiatrie de liaison du CHUV offre des services importants de debriefing et de suivi psychiatrique qui sont difficilement réalisables dans une autre langue que la langue maternelle. Les séjours des patients peuvent être très longs, dépassant parfois les 100 jours. Il est donc important d'offrir des traitements à proximité du domicile pour faciliter les déplacements des proches dont le soutien est essentiel.

\section{Figure 3}

Trajectoire du patient brûlé dans le réseau. Le patient est trié lors de son admission et orienté en fonction de sa gravité vers la filière hospitalière, ou vers un traitement ambulatoire

Abréviations: ambul = ambulatoire; SIP = soins intensifs de pédiatrie; réhabil = réhabilitation.

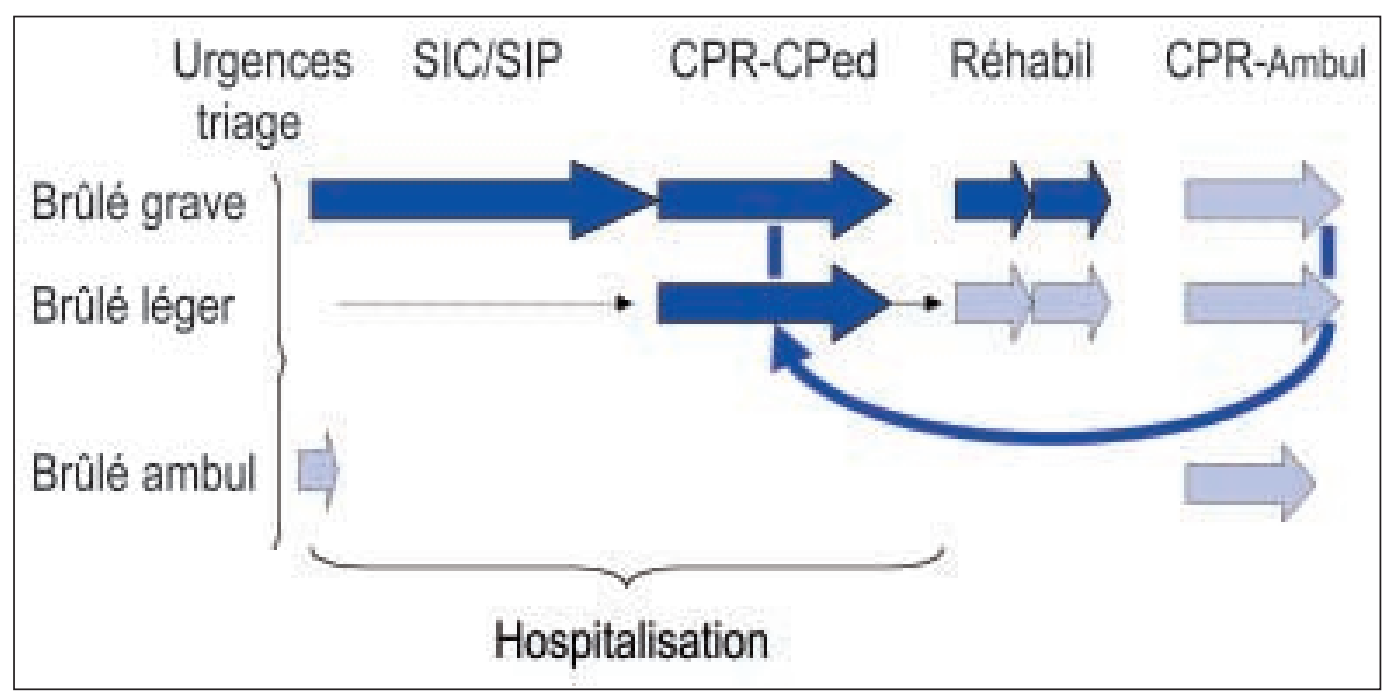




\section{Caractéristiques du CRGB et relations avec le réseau de soins Romand}

Le CRGB se compose d'une unité intégrée dans les soins intensifs chirurgicaux, du service de CPR et de sa consultation ambulatoire. Il fonctionne depuis plusieurs années en réseau avec l'Hôpital Universitaire de Genève et les hôpitaux romands. Les grands brûlés sont directement référés au CRGB, ou après triage initial dans les hôpitaux périphériques. Des critères d'admission basés sur ceux l'ABA (www.ameriburn.org) (tab. 2), ont été diffusés en 1998 dans l'ensemble de ces hôpitaux ainsi qu'à la REGA. Lorsque que le CRGB est débordé, il sollicite la collaboration du Centre de Zurich et organise le transfert des patients. Il arrive cependant que les 2 centres soient saturés, ce qui pose de sérieux problèmes de logistique et de triage.

Selon la gravité de leurs brûlures, les patients ont un parcours variable (fig. 3). Les grands brûlés font des séjours prolongés dans les soins intensifs dédiés (p.ex. un minimum de 2 mois de soins intensifs est prévisible pour une brûlure de $50 \%$ BSA), avant le transfert en division.

La réhabilitation initiale et ambulatoire est effectuée par l'équipe de physiothérapie du CHUV. Celle-ci a diffusé une formation spécifique de physiothérapie dans les villes et cantons voisins pour permettre un véritable traitement ambulatoire des brûlés. Le Centre médical de Lavey-les-Bains assure depuis peu la réhabilitation spécialisée. Plusieurs patients romands y ont été traités en 2002 et 2003, dans des installations modernes et performantes. Une collaboration est en discussion avec la Clinique Romande de Réhabilitation de la SUVA à Sion. Pour la phase tardive, soit pendant les 2 à 3 années qui suivent l'accident, il est souvent nécessaire d'effectuer des corrections chirurgicales de cicatrice et des appareillages. Ces interventions sont effectuées en grande partie au CHUV.

Sur le plan social, une association de patients brûlés «Flavie» (www.flavie.ch) a été créée en 2002 sous l'impulsion des soignants du CHUV, qui fournit un soutient social et un service d'écoute et d'entraide aux patients victimes des brûlures. Elle prévoit de défendre leurs intérêts et collaborer aux projets de prévention et d'information. Cette association fait partie des 5 membres fondateurs de l'association européenne des brûlés.

L'ensemble de ce dispositif permet d'assurer aux brûlés graves de notre région un traitement médico-chirurgical complet, allant de l'admission en urgence, jusqu'à la réhabilitation, sans oublier la ré-intégration sociale.
Tous les patients Suisses répondant aux critères de l'ABA ne sont pas traités dans les 2 centres reconnus. En 1998, une estimation basée sur les codes VESKA des hospitalisations du canton de Vaud, a permis d'identifier un total de 102 patients brûlés: 65 avaient été traités au CHUV, alors que 37 patients avaient été traités dans d'autres hôpitaux du Canton: 12 d'entre eux avaient requis de la chirurgie [1]. En d'autres termes, une partie des patients brûlés justifiant d'une prise en charge spécialisée n'en bénéficie pas pour différentes raisons y compris la surcharge des 2 centres, ce qui mériterait un débat.

\section{Aspects économiques et politiques}

En une période où l'accroissement des coûts hospitaliers doit être maîtrisé, la prise en charge économique des brûlés par 2 cantons grève lourdement leurs dépenses de santé. En effet le traitement des brûlés graves est coûteux. En phase initiale, un grand brûlé coûte entre Fr. 4000.- et 6000.- par jour: le traitement d'un brûlé de plus de $50 \%$ BSA excède les Fr. 150000.-, particulièrement si l'on intègre la phase de réhabilitation. Or les accidents par brûlures sont un problème de santé publique. Fournir à la population ce type de traitements est une nécessité et doit être analysée en terme de «service». Maintenir et soutenir nos 2 centres est une question de politique de santé d'importance nationale, qui doit être discutée au niveau de la Conférence des Directeurs sanitaires, voire au niveau de l'Office fédéral de la santé publique: les prestations offertes devraient correspondre à des standards internationaux, qui ne peuvent être garantis que par des centres universitaires. A l'instar de la transplantation d'organes, un financement fédéral permettrait à la fois un meilleur contrôle des dépenses et une répartition plus équitable de la charge économique.

\section{Conclusion}

Le traitement des brûlés a considérablement évolué au cours de la dernière décennie et la mortalité à diminué. Les traitements chirurgicaux ont progressé avec l'apparition de nouveaux substituts cutanées, mais aussi avec le développement des cultures cellulaires multicouches, améliorant le devenir fonctionnel et la réinsertion sociale des patients. Ces progrès importants ont pu être réalisés grâce au traitement en centre spécialisé à Lausanne et à Zurich, et aux compétences spécifiques qui y sont centralisées. Deux centres de 
brûlés sont justifiés pour une population de la taille de la Suisse sur la base des critères internationaux. Les caractéristiques linguistiques et géographiques de notre pays sont des justifications psycho-sociales supplémentaires à l'existence de ces 2 centres.

L'importance du centre romand a été reconnu par l'association Vaud-Genève au début de 2003, et le CHUV va prendre des dispositions pour le rendre plus opérationnel, et mieux intégré au plateau technique. Nos centres fonctionnent actuellement en flux tendu. Les récents événements et la menace terroriste chronique démontrent à quel point le nombre de places est critique. Il est nécessaire, non seulement de maintenir ces lits spécialisés, mais également de disposer d'une capacité de réserve.

\section{Références}

1 Von Bremen K, Wasserfallen JB. Evaluation médico-économique des grands brûlés. Rapport pour la Collaboration Vaud-Genève. Lausanne: Direction médicale du CHUV; 1999.

2 Munster AM. The 1996 Presidential address Burns of the World. J Burn Care Rehab 1996;17:477-84.

3 Linn BS, Stephenson SE, Bergstresser PR, Smith J. Are burn units the best place to treat burn patients? J Surg Res 1977;23:1-5.
4 Sheridan RL. Burn care: results of technical and organizational progress. JAMA 2003;290:719-22.

5 Krupp S. Réflexion sur l'organisation d'un centre des brûlés. Bull Méd Suisses 1986;67:1612-4.

6 Becker DS. Toxic epidermal necrolysis. Lancet 1998;351:1417-20.

7 Sheridan RL, Weber JM, Schulz JT, Ryan CM, Low HM, Tompkins RG. Management of severe toxic epidermal necrolysis in children. J Burn Care Rehab 1999;20(6):497-500.

8 Vernez M, Raffoul W, Gailloud-Matthieu M-C, Efloff D, Senechaud I, Panizzon RG, Benathan M. Quantitative assessment of cell viability and apoptosis in cultured epidermal autografts: application to burn therapy. Int J Artif Organs 2003;26:793-803.

9 Berger MM, Chioléro R. Réanimation initiale du grand brûlés adulte. Rev Méd Suisse Romande 1998;118:107-15.

10 Berger MM, Bernath MA, Chioléro RL. Resuscitation, anaesthesia and analgesia of the burned patient. Curr Opin Anaesthesiol 2001;14:431-43.

11 Berger MM, Chioléro R. Support métabolique et nutritionnel du patient brûlé adulte. Rev Méd Suisse Romande 1998;118:127-32.

12 Favarger N, Chioléro R, Berger M, Egloff DV. Pourquoi un centre des brûlés au CHUV? Rev Méd Suisse Romande 1998;118:103-5

13 Berger MM, Revelly JP, Chioléro R. Informatisation d'une unité de soins intensifs: expérience de la phase pilote au CHUV. Point de vue médical. Méd Hyg 1999;57:2152-215. 